\title{
ON EUCLIDEAN LOCAL GROUPS SATISFYING CERTAIN CONDITIONS
}

\author{
MASATAKE KURANISHI
}

Let $G$ be a euclidean local group, that is, a topological local group the space of which is homeomorphic to a euclidean space. The purpose of this note is to prove the following theorem:

Theorem. Let $G$ have a neighborhood $U$ of identity $e$, in which a metric $\rho(x, y)$ can be introduced satisfying the following conditions:

(A) If $x, y, x y \in U, 1$ then $K_{2} \rho(y, e) \leqq \rho(x y, x) \leqq K_{1} \rho(y, e)$.

(B) If $x, x^{2}, \cdots, x^{2^{n-1}}, x^{2^{n}} \in U, y, y^{2}, \cdots, y^{2^{n-1}}, y^{2^{n}} \in U$, then $K_{4} 2^{n} \rho(x, y) \leqq \rho\left(x^{2^{n}}, y^{2^{n}}\right) \leqq K_{3} 2^{n} \rho(x, y)$, with positive constants $K_{i}, i=1$, $2,3,4$.

Then $G$ is a local Lie group, and vice versa.

$P$. A. Smith [2] $]^{2}$ has obtained a necessary and sufficient condition for $G$ to be a local Lie group. He says that if we can introduce into a neighborhood of $e$ a coordinate system $a^{1}, \cdots, a^{r}$, with respect to which the product function $a b$ is expressible in the form, written vectorially,

$$
a b=a+b+|a| F(a, b)
$$

where $|a|=\left(\sum\left(a^{i}\right)^{2}\right)^{1 / 2}$ and where $F$ satisfies the sole condition that $F \rightarrow 0$ as $a \rightarrow e, b \rightarrow e$, then $G$ is a local Lie group and vice versa. A coordinate system satisfying this condition is called by him (right) regular. It is shown $[1 ; 2]$ that a coordinate system in which the product function $a b$ is of class $C^{1}$ with respect to $b$ fixing $a$ is regular and that the euclidean metric of a regular coordinate system satisfies our conditions (A) and (B).

Our assumptions (A) and (B) are mainly used to select a uniformly convergent subsequence from the function family $P_{n}(x, y)$ $=\left(x^{1 / 2^{n}} \cdot y^{1 / 2^{n}}\right) 2^{n}, n=1,2, \cdots$, which, in some sense, corresponds to differentiating the product function at the identity $e$.

The author is indebted to Prof. T. Nakayama for his kind encouragement and advice.

LEMMA 1. Let $U$ be a neighborhood of e in $G$ which contains no sub-

Received by the editors March 4, 1949.

1 In details, we must write-instead of $x y \in U-x y$ exists and is contained in $U$. But we shall of ten omit "exists" in such a case in the following for simplicity.

2 Numbers in brackets refer to the references cited at the end of the paper. 
group in the large except $\{e\}$. Then there exists a neighborhood $V$ of $e$ such that $x, y, x^{2}=y^{2} \in V$ implies $x=y$.

Proof. ${ }^{2}$ We can assume without loss of generality that $\bar{U}$ is compact and for any arbitrary three elements $x, y, z$ of $U$ the products $(x y) z$ and $x(y z)$ exist. Take $W$ such that $U \supset W^{4}, W=W^{-1}$. As $\bar{U}$ is compact, there exists a neighborhood $V$ of $e$ such that for any $g \in \bar{U}$, we have $g^{-1} V g \subset W$. Now if $x, y, x^{2}=y^{2} \in V$, put $x^{-1} y=a$. Then $a x a=x, a \in V^{2} \subset W \subset U$, and it follows that if $a, a^{2}, \cdots, a^{m} \in U$, then $a^{2 m}=x^{-1} a^{-m} x a^{m} \in x^{-1} W \subset W^{2} \subset U, \quad a^{2 m+1}=a^{2 m} \cdot a \in W^{2} W^{2} \subset U$. This means that for any integer $n, a^{n} \in U$; consequently from the assumption $a=e, x=y$.

REMARK. In Lemma 1 the assumption that $G$ is euclidean is not necessary. Let $\sigma$ be a mapping $y \rightarrow y^{2}$. If $G$ is euclidean and if $U$ is sufficiently small, then $\sigma(U)$ is an open subset of $G$ and $\sigma$ is a homeomorphism between $U$ and $\sigma(U)$. The proof is clear from the Brouwer's theorem on the invariance of domain and from the fact that $\bar{U}$ is compact and that $\sigma$ is one-to-one.

LEMMA 2. Let $G$ have a neighborhood $U$ of $e$ such that for any element $y$ of $U-\{e\}$ there exists an integer $n$ satisfying $y^{2^{n}} \notin \bar{U}$. Then, if $V$ is a sufficiently small neighborhood of $e$, we can construct a real-valued continuous function $f(y)$ defined on $V$ satisfying the following conditions:

(i) If $y, y^{2} \in V$, then $f\left(y^{2}\right) \geqq f(y)$.

(ii) $f(y)=0$ if and only if $y=e$ provided $y \in V$.

Proof. If $y, y^{2}, y^{2^{2}}, \cdots, y^{2^{n}} \in \bar{V}, y^{2^{n+1}} \in \bar{V}$, we put $n=\delta_{V}(y)$. Take an element $p$ of $V$, we note that if $k \leqq \delta_{V}(p)$, then $p \neq p^{2}$. Consequently if we take a sufficiently small neighborhood $W$ of $e$, then for any $i<j \leqq \delta_{V}(p)$, we have $\sigma^{i}(W p) \cap \sigma^{i}(W p)=\Theta, \sigma^{\delta}(p)+1(W p)$ $\cap \bar{V}=\Theta$, where $\sigma^{0}(y)=y, \sigma^{2}(y)=y^{2}, \sigma^{i+1}(y)=\sigma^{1}\left(\sigma^{i}(y)\right)$.

Construct a continuous function $f_{p}^{0}(y)$ defined on $V$ such that

$$
0 \leqq f_{p}^{0}(y) \leqq 1 \text {; if } y \notin W p \text {, then } f_{p}^{0}(y)=0 \text {; and } f_{p}^{0}(p)=1 \text {. }
$$

For any integer $k \leqq \delta_{V}(p)$ put

$$
\begin{array}{ll}
\text { if } y \notin \sigma^{k}(W p), & f_{p}^{k}(y)=0, \\
\text { if } y \in \sigma^{k}(W p), & f_{p}^{k}(y)=f_{p}^{0}\left(y^{1 / 2^{k}}\right), \\
\end{array}
$$

If $V$ is sufficiently small, by the remark to Lemma $1, f_{p}^{k}(y)$ is a continuous function defined on $V$. Put

- This simple proof was suggested to the author by T. Hayashida. The author's original proof is more complicated. 


$$
f_{p}(y)=\sum_{k=0}^{\delta_{\mathrm{V}}(p)} f_{p}^{k}(y)
$$

It is easy to see that $f_{p}(y)$ is a continuous function defined on $V$ and $0 \leqq f_{p}(y) \leqq 1, f_{p}(p)=1, f_{p}\left(y^{2}\right) \geqq f_{p}(y)$. As $\cup_{p \in-V(e)} V_{p}=V-\{e\}$, where $V_{p}=\left\{y ; f_{p}(y)>0\right\}$, we can select a countable set $\left\{p_{n}\right\}$ such that $\bigcap_{n=1}^{\infty} V_{p_{n}}=V-\{e\}$. Put

$$
f(y)=\sum_{n=1}^{\infty} \frac{1}{2^{n}} f_{p_{n}}(y) .
$$

It is clear that $f(y)$ satisfies our conditions (i) and (ii).

Lemma 3. Under the same assumption as in Lemma 2, there exists a neighborhood $W$ of e such that for an arbitrary element $x$ of $W, W$ contains a unique element $x^{1 / 2}$ satisfying $\left(x^{1 / 2}\right)^{2}=x$.

Proof. Take a sufficiently small neighborhood $V$ of $e$, where we can construct the function $f(y)$ as stated in Lemma 2. Put $V$. $=\{y ; f(y)<\epsilon\}$. If $V$ is sufficiently small, by the Remark to Lemma 1 , $\sigma(V)$ is an open set. From the condition (ii) which $f(y)$ satisfies, there exists $\epsilon>0, V_{\epsilon} \subset \sigma(V) \cap V$. This means that for any element $y$ of $V$. there exists $y^{1 / 2} \in V$ such that $\left(y^{1 / 2}\right)^{2}=y$. But since $f\left(y^{1 / 2}\right) \leqq f(y)<\epsilon$, $y^{1 / 2} \in V_{\text {c. }}$. The uniqueness of $y^{1 / 2}$ has already been proved in Lemma 1 .

By Lemma 3, there exists $x^{1 / 2^{n}}$ such that $\left(x^{1 / 2^{n}}\right)^{2^{n}}=x$ and $x^{1 / 2^{n}} \in W$ for any element $x$ of $W$. We can assume without loss of generality $W \subset U$, where $U$ is a neighborhood of $e$ satisfying the assumption of our theorem. We shall write $|x|$ instead of $\rho(x, e)$. Then by (B), $\left|x^{1 / 2^{n}}\right| \leqq\left(1 / K_{4}\right)\left(1 / 2^{n}\right)|x|$. We can prove, by induction, the existence of $\left(x^{1 / 2^{n}}\right)^{m}$ and

$$
\left|\left(x^{1 / 2^{n}}\right)^{m}\right| \leqq \frac{K_{1}}{K_{4}} \frac{m}{2^{n}}|x|
$$

for any arbitrary integer $m \leqq 2^{n}$. Assume, in fact, $\left|\left(x^{1 / 2^{n}}\right)^{m}\right|$ $\leqq\left(K_{1} / K_{4}\right)\left(m / 2^{n}\right)|x|$ for some $m<2^{n}$, then

$$
\begin{aligned}
\left|\left(x^{1 / 2^{n}}\right)^{m+1}\right| & =\left|\left(x^{1 / 2^{n}}\right)^{m} x^{1 / 2^{n}}\right| \leqq K_{1}\left|x^{1 / 2^{n}}\right|+\left|\left(x^{1 / 2^{n}}\right)^{m}\right| \\
& \leqq \frac{K_{1}}{K_{4}} \frac{1}{2^{n}}|x|+\frac{K_{1}}{K_{4}} \frac{m}{2^{n}}|x| \\
& =\frac{K_{1}}{K_{4}} \frac{m+1}{2^{n}}|x|,
\end{aligned}
$$

as we can see easily from (A) that 


$$
|x y| \leqq K_{1}|y|+|x| \text {. }
$$

By using the uniqueness of $x^{1 / 2}$, it is easy to see that $m / 2^{n}=m^{\prime} / 2^{n^{\prime}}$ implies $\left(x^{1 / 2^{n}}\right)^{m}=\left(x^{1 / 2^{n}}\right)^{m^{\prime}}$. Now we put $f\left(m / 2^{n}\right)=\left(x^{1 / 2^{n}}\right)^{m}$; then $f\left(m / 2^{n}\right) f\left(m^{\prime} / 2^{n^{\prime}}\right)=f\left(m / 2^{n}+m^{\prime} / 2^{n^{\prime}}\right)$, and from these and from the inequality $\left({ }^{*}\right)$ follow the uniform continuity of the function $f$ on the set $\left\{m / 2^{n} ; m \leqq 2^{n}\right\}$, which is everywhere dense in the interval $[0,1]$. Therefore $f$ can be extended to a continuous function $\bar{f}$ defined on $[0,1]$. If we put $x^{\lambda}=f(\lambda), x^{-\lambda}=\left(x^{-1}\right)^{\lambda}$, for any $0 \leqq \lambda \leqq 1$, then $\left|x^{\lambda}\right|$ $\leqq\left(K_{1} / K_{4}\right)|x|$ and $x^{\lambda} \cdot x^{\mu}=x^{\lambda+\mu}$ if both sides have meaning. This proves the following lemma.

Lemma 4. Under the same assumption as in the theorem, there exist neighborhoods $W_{1}$ and $W_{2}$ of e such that for any element $x$ of $W_{1}$, there exists a unique one-parameter subgroup $x^{\lambda}$ contained in $W_{2}$.

From (A),

$\left(\mathrm{D}^{\prime}\right) \quad \rho(a x, a y) \leqq K_{1} \rho\left((a y)^{-1} \cdot a x, e\right)=K_{1} \rho\left(y^{-1} \cdot x, e\right) \leqq \frac{K_{1}}{K_{2}} \rho(x, y)$.

Take a sufficiently small neighborhood $V$ of $e$, and put

$$
\begin{array}{ll}
\Sigma=\left\{x ; x \in V, X^{2} \notin V\right\}, & C=\min _{x \in \Sigma}|x|, \\
\Omega=\left\{w ; w=a^{-1} x a, x \in \Sigma, a \in V\right\}, & D=\max _{x \in \mathbf{\Omega}}|x| .
\end{array}
$$

Then $\bar{\Sigma} \boxplus e, C>0, D>0$, and

$$
\left|a^{-1} x a\right| \leqq \frac{D}{C}|x| \quad \text { for } x \in \Sigma, a \in V .
$$

Take $y \in V$, then for some integer $n, y^{2^{n}} \in \Sigma$ and for every integer $0 \leqq m \leqq n, y^{2 m} \in V$. From (B) and (**)

$$
\begin{aligned}
\left|a^{-1} y a\right| & \leqq \frac{1}{2^{n}} \frac{1}{K_{4}}\left|a^{-1} y^{2^{n}} a\right| \leqq \frac{1}{2^{n}} \frac{1}{K_{4}} \frac{D}{C}\left|y^{2^{n}}\right| \leqq \frac{K_{8} D}{K_{4} C}|y|, \\
\left(D^{\prime \prime}\right) \quad \rho(x a, y a) & \leqq K_{1} \rho\left(a^{-1} y^{-1} x a, e\right) \leqq \frac{K_{1} K_{3} D}{K_{4} C} \rho\left(y^{-1} x, e\right) \\
& \leqq \frac{K_{1} K_{3} D}{K_{2} K_{4} C} \rho(x, y),
\end{aligned}
$$

if $x, y, a$ is sufficiently near to $e$.

From $\left(D^{\prime}\right)$ and $\left(D^{\prime \prime}\right)$ we can see that for a sufficiently small neigh- 
borhood $V$ of $e$, there exists $A>0$ such that

(D) if $x, y, x^{\prime}, y^{\prime} \in \bar{V}, \rho\left(x y, x^{\prime} y^{\prime}\right) \leqq A\left\{\rho\left(x, x^{\prime}\right)+\rho\left(y, y^{\prime}\right)\right\}$.

If we take a sufficiently small neighborhood $V$ of $e$, from (C), (B), and (D) we can easily prove the existence of $P_{n}(x, y)=\left(x^{1 / 2^{n}} y^{1 / 2^{n}}\right)^{2^{n}}$ and

$$
\begin{aligned}
\left|P_{n}(x, y)\right|=\left|\left(x^{1 / 2^{n}} y^{1 / 2^{n}}\right) 2^{2^{n}}\right| & \leqq \frac{K_{1} K_{3}}{K_{4}}(|x|+|y|), \\
\rho\left(P_{n}(x, y), P_{n}\left(x^{\prime}, y^{\prime}\right)\right) & \leqq \frac{K_{8} A}{K_{4}}\left\{\rho\left(x, x^{\prime}\right)+\rho\left(y, y^{\prime}\right)\right\}
\end{aligned}
$$

for arbitrary $x, y, x^{\prime}, y^{\prime} \in \bar{V} \subset U$.

Therefore $P_{n}(x, y), n=0,1,2, \cdots$, is an equi-continuous and uniformly bounded family of functions defined on $\bar{V} \times \bar{V}$, and consequently a uniformly convergent subsequence $\left\{P_{n^{\prime}}(x, y)\right\}$ can be selected from $\left\{P_{n}(x, y)\right\}$. Put $P(x, y)=\lim _{n^{\prime} \rightarrow \infty} P_{n^{\prime}}(x, y)$.

Now we shall prove that if we define the product $x \circ y=P(x, y), V$ becomes an abelian local Lie group. We denote this local group by $H$.

(1) Commutativity is clear from $\left(x^{1 / 2^{n}} y^{1 / 2^{n}}\right)^{2^{n}}=x^{1 / 2^{n}}\left(y^{1 / 2^{n}} x^{1 / 2^{n}}\right)^{2^{n}}$ $\left(x^{1 / 2^{n}}\right)^{-1}$.

(2) Associative law: Put $P(x, y)=\epsilon_{n^{\prime}} P_{n^{\prime}}(x, y)$, then $\lim _{n^{\prime} \rightarrow \infty} \epsilon_{n^{\prime}}=e$. Using (D), (E), and (F),

$$
\rho\left(P_{n^{\prime}}(P(x, y), w), P_{n^{\prime}}\left(P_{n^{\prime}}(x, y), w\right)\right) \leqq \frac{K_{8} A^{2}}{K_{4}} \rho\left(\epsilon_{n^{\prime}}, e\right),
$$

from the definition $P_{n^{\prime}}\left(P_{n^{\prime}}(x, y), w\right)=P_{n^{\prime}}\left(x, P_{n^{\prime}}(y, w)\right)$. From these we can see $(x \circ y) \circ w=x \circ(y \circ w)$.

(3) $x^{\lambda} \circ x^{\mu}=x^{\lambda+\mu}$, which shows the existence of the inverse, and that every one-parameter subgroup of $G$ is also that of $H$.

(4) As $H$ is abelian, we can find easily $x_{1}, x_{2}, \cdots, x_{r}$ of $H$ (where $r$ is the dimension of $G)$ such that $W=\left\{y ; y=x_{1}^{\lambda_{1}} \circ x_{2}^{\lambda_{2}} \circ \cdots \circ x_{r}^{\lambda_{r}}\right.$, $\left.\left|\lambda_{i}\right| \leqq 1, i=1,2, \cdots, r\right\}$ is a neighborhood of $e$ and mapping $\left(\lambda_{1}, \lambda_{2}, \cdots, \lambda_{r}\right) \rightarrow x_{1}^{\lambda_{1}} \circ x_{2}^{\lambda_{2}} \circ \cdots \circ x_{r}^{\lambda_{r}}$ is topological. Moreover

$$
\left(x_{1}^{\lambda_{1}} \circ \cdots \circ x_{r}^{\lambda_{1}}\right) \circ\left(x_{1}^{\lambda_{1}^{\prime}} \circ \cdots \circ x_{r}^{\lambda_{r}^{\prime}}\right)=\left(x_{1}^{\lambda_{1}+\lambda_{1}^{\prime}} \circ \cdots \circ x_{r}^{\lambda_{r}+\lambda_{r}^{\prime}}\right) .
$$

Thus we can see that $H$ is a Lie group.

LEMMA 5. Under the same assumptions as in the theorem the local group consisting of inner automorphisms of $G$ is a linear group.

Proof. From the uniqueness of $x^{1 / 2}$, it is easy to see $p x^{1 / 2} p^{-1}$

- The proof of this inequality is essentially due to P. A. Smith [2]. 
$=\left(p x p^{-1}\right)^{1 / 2}$, for arbitrary $x, y, p \in V$. From this and from the sufficient smallness of $\left|P_{n}(x, y)\right|$, we can deduce immediately that for arbitrary $p, x, y \in V, P_{n}\left(p^{-1} x p, p^{-1} y p\right)=p^{-1} \cdot P_{n}(x, y) p$, which implies $\left(p^{-1} x p\right) \circ\left(p^{-1} y p\right)=p^{-1}(x \circ y) p$. Thus $x \rightarrow p^{-1} x p$ is an automorphism of the abelian Lie group $H$, which proves the lemma.

Leмma $6 .^{5}$ Let $N$ be a closed local normal subgroup of $G$, satisfying the following conditions:

(1) $N$ is an abelian local Lie group,

(2) $G / N$ is a local Lie group,

(3) there exists a set $M$ such that for any a sufficiently near to e, $\mathrm{Na}$ contains one and only one element $a^{\prime}$ of $M$ depending continuously on $G / N$.

If $G$ contains $N$ satisfying the above conditions, $G$ is a local Lie group.

Proof. As $N$ is an abelian Lie group, we can introduce a coordinate system $u\left(\xi^{1}, \cdots, \xi^{n}\right)$ in $N$ satisfying $u\left(\xi^{1}, \cdots, \xi^{n}\right)$ $\cdot u\left(\xi^{\prime 1}, \cdots, \xi^{\prime n}\right)=u\left(\xi^{1}+\xi^{\prime 1}, \cdots, \xi^{n}+\xi^{\prime n}\right)$. The transformation by an element $a$ of $G$ induces an automorphism in $N$, given by

$$
\text { au } \begin{aligned}
\left(\xi^{1}, \cdots, \xi^{n}\right) a^{-1} & =u\left(\xi^{\prime 1}, \cdots, \xi^{\prime n}\right), \\
\left(\xi^{\prime 1}, \cdots, \xi^{\prime n}\right) & =\left(\xi^{1}, \cdots, \xi^{n}\right) A_{a},
\end{aligned}
$$

where $\left(A^{\alpha_{i}^{j}}\right)$ is a real matrix of degree $n$. As $G=N M$, we can put for arbitrary elements $p, q \in M$ sufficiently near to $e$,

$$
\begin{aligned}
p q & =\Psi(p, q)(p \circ q), \\
\Psi(p, q) & =u\left(\Psi^{1}(p, q), \cdots, \Psi^{n}(p, q)\right) \in N, \quad p \circ q \in M,
\end{aligned}
$$

where, by (3), both $p \circ q$ and $\Psi(p, q)$ are continuous functions on $M \times M$. Then by the product $p \circ q, M$ is a local Lie group isomorphic with $G / N$, and $M \ni p \rightarrow A_{p}$ is a representation of the local Lie group $M$. Consequently, introducing the canonical coordinate $v\left(\eta^{1}, \cdots, \eta^{m}\right)$ in $M, A_{\nabla}\left(\eta^{1}, \cdots, \eta^{m}\right)_{j}^{\}}$are analytic functions of $\left(\eta^{1}, \cdots, \eta^{m}\right)$.

From the associative law of the product of $G$, if $p, q, r$ are sufficiently near to $e$, we can see easily that

(1) $\Psi^{i}(p, q)+\Psi^{i}(p \circ q, r)=\sum_{j=1}^{n} A_{p j}^{i} \Psi^{j}(q, r)+\Psi^{i}(p, q \circ r), i=1, \cdots, n$.

Let $\phi(p)$ be an arbitrary continuous function defined on $M$ and

- In the proof of this lemma, the fact that the space of $G$ is euclidean is not used. To prove our theorem we need only the case when $N$ is the center of $G$. But we state the lemma in this general form, because this lemma is applicable to some theorem on locally compact groups (cf. [3]). 
taking values in $N . M_{\phi}=\left\{p_{\phi} ; p_{\phi}=\phi(p) p, p \in M\right\}$ also satisfies the condition (3), if $M$ is replaced by $M_{\phi}$. We can put

$$
\begin{gathered}
p_{\phi} \cdot q_{\phi}=\Psi_{\phi}(p, q)\left(p_{\phi} \circ q_{\phi}\right), \\
\Psi_{\phi}(p, q) \in N, \quad p_{\phi} \circ q_{\phi} \in M_{\phi} .
\end{gathered}
$$

As $p_{\phi} \circ q_{\phi}=(p \circ q)_{\phi}=\phi(p \circ q)(p \circ q)$, the relation between $\Psi^{i}$ and $\Psi_{\phi}^{\prime \prime}$ is given by

(2) $\Psi_{\phi}^{i}(p, q)=\phi^{i}(p)+\sum_{j=1}^{n} A_{p j}^{i} \phi^{i}(q)+\Psi^{i}(p, q)-\phi^{i}(p \circ q), i=1,2, \cdots, n$.

Take two sufficiently small numbers $\alpha$ and $\beta$ such that $U$ $=\left\{v\left(\eta^{1}, \cdots, \eta^{m}\right) ;\left|\eta^{i}\right|<\alpha\right\}$ and $V=\left\{v\left(\eta^{1}, \cdots, \eta^{m}\right) ;\left|\eta^{i}\right|<\beta\right\}$ satisfy $U \supset V \circ V \circ V$ and such that if $p, q, r \in U$, equalities (1) and (2) have meanings. Take a real function $c(p)$ defined on $U$ such that $c\left(v\left(\eta^{1}, \cdots, \eta^{m}\right)\right)$ is of class $C^{3}$ with respect to $\eta^{1}, \cdots, \eta^{m}$ and which satisfies the following conditions:

If $p \in V$, then $c(p)=0$,

$$
\int_{V} c\left(v\left(\eta^{1}, \cdots, \eta^{m}\right)\right) d \eta^{1} \cdots d \eta^{m}=\int_{V} c(v(\eta)) d \eta=\int_{U} c(v(\eta)) d \eta=1 .
$$

Put

$$
\phi_{0}^{i}(p)=-\int_{V} \Psi^{i}(p, v(\eta)) c(v(\eta)) d \eta=-\int_{U} \Psi^{i}(p, v(\eta)) c(v(\eta)) d \eta
$$

and $\phi_{0}(p)=u\left(\phi_{0}^{1}(p), \cdots, \phi_{0}^{n}(p)\right) \in N$. Then from (1) and (2), if $p, q \in V$

$$
\begin{array}{rlrl}
\Psi_{\phi_{0}}^{i}(p, q) & =\int_{\nabla} \Psi^{i}(p, q \circ v(\eta)) c(v(\eta)) d \eta-\int_{V} \Psi^{i}(p, v(\eta)) c(v(\eta)) d \eta \\
& =F_{1}(p, q)-F_{2}(p), & i=1,2, \cdots, n .
\end{array}
$$

Put $q \circ v\left(\eta^{1}, \cdots, \eta^{m}\right)=v\left(\eta^{\prime 1}, \cdots, \eta^{\prime m}\right)$, and changing the integral variable $\eta$ by $\eta^{\prime}$, we can see

$$
\begin{aligned}
F_{1}^{i}(p, q) & =\int_{V} \Psi^{i}(p, q \circ v(\eta)) c(v(\eta)) d \eta \\
& =\int_{q \circ V} \Psi^{i}\left(p, v\left(\eta^{\prime}\right)\right) c\left(q^{-1} \circ v\left(\eta^{\prime}\right)\right) J\left(\eta^{\prime}\right) d \eta^{\prime} \\
& =\int_{U} \Psi^{i}\left(p, v\left(\eta^{\prime}\right)\right) c\left(q^{-1} \circ v\left(\eta^{\prime}\right)\right) J\left(\eta^{\prime}\right) d \eta^{\prime},
\end{aligned}
$$


because $\eta \rightarrow \eta^{\prime}$ is an analytic function of $\eta$ and $q \circ V \subset U$. As $c\left(v\left(\eta^{\prime}\right)\right)$, $q \circ r$, and $q^{-1}$ are functions of class $C^{3}$, the above equality shows that $F_{1}^{\prime}(p, v(\eta))$ are functions of class $C^{3}$ with respect to $\eta$ when $p$ is fixed. Consequently, by $(3), \Psi_{\phi_{0}}^{s}(p, v(\eta))$ are functions of class $C^{3}$ with respect to $\eta$ when $p$ is fixed. From (1), we can get

$$
\begin{aligned}
\sum_{j=1}^{n}\left\{\int_{U} A_{p j}^{i} c(p) d p\right\} & \Psi^{i}(q, r) \\
= & \int_{U} \Psi^{i}(p, q) c(p) d p+\int_{U} \Psi^{i}(p \circ q, r) c(p) d p \\
& -\int_{U} \Psi^{i}(p, q \circ r) c(p) d p .
\end{aligned}
$$

From the fact that if $U$ is sufficiently small, $A_{p}(p \in U)$ is sufficiently near to the identity matrix and that $\int_{U} c(p) d p=1$, it follows that the determinant $\left|\int_{U} A_{p j} c(p) d p\right|$ is not zero. Consequently, using the same argument as above on $\int_{U} \Psi^{i}(p \circ q, r) c(p) d p$, we can deduce from (4) that if $\Psi^{i}(p, q)$ is of class $C^{3}$ with respect to $q$ when $p$ is fixed, $\Psi^{i}(p, q)$ is of class $C^{3}$ with respect to $p$ when $q$ is fixed. Thus we have proved that $\Psi_{\phi_{0}}^{\prime}(p, q)$ is of class $C^{3}$ with respect to both $p$ and $q$ in the coordinate system $v\left(\eta^{1}, \cdots, \eta^{m}\right)$. As any element $p$ of $G$ is written uniquely as $p=n m_{\phi}, n \in N, m_{\phi} \in M$, we can introduce a coordinate system $w\left(\zeta^{1}, \cdots, \zeta^{n+m}\right)$ in $G$ by

$$
\begin{aligned}
& w\left(\zeta^{1}, \cdots, \zeta^{n+m}\right) \\
& \quad=u\left(\zeta^{1}, \cdots, \zeta^{n}\right) \phi_{0}\left(v\left(\zeta^{n+1}, \cdots, \zeta^{n+m}\right)\right) v\left(\zeta^{n+1}, \cdots, \zeta^{n+m}\right) .
\end{aligned}
$$

As $\phi_{0}(v(\eta)) \in N$ and $N$ is abelian, we can easily verify that the product function $f^{i}\left(\zeta^{1}, \cdots, \zeta^{n+m} ; \zeta^{\prime 1}, \cdots, \zeta^{\prime n+m}\right)(i=1,2, \cdots, n+m)$ of $G$ with respect to this coordinate system is given by

$$
\begin{aligned}
& f^{i}\left(\zeta^{1}, \cdots, \zeta^{n+m} ; \zeta^{\prime 1}, \cdots, \zeta^{n+m}\right)=\zeta^{i}+A_{v\left(\zeta^{n+1}, \cdots, \zeta^{n+m}\right) j}^{i} \zeta^{i} \\
& +\Psi_{\phi_{0}}^{i}\left(v\left(\zeta^{n+1}, \cdots, \zeta^{n+m}\right), v\left(\zeta^{\prime n+1}, \cdots, \zeta^{n+m}\right)\right) \\
& (i=1,2, \cdots, n), \\
& f^{i}\left(\zeta^{1}, \cdots, \zeta^{n+m} ; \zeta^{\prime 1}, \cdots, \zeta^{\prime n+m}\right) \\
& =g^{i}\left(\zeta^{n+1}, \cdots, \zeta^{n+m} ; \zeta^{\prime n+1}, \cdots, \zeta^{\prime n+m}\right) \\
& (i=n+1, \cdots, n+m) \text {, }
\end{aligned}
$$

where $g^{i-n}\left(\zeta^{n+1}, \cdots, \zeta^{n+m} ; \zeta^{\prime n+1}, \cdots, \zeta^{\prime n+m}\right)$ is the product function of $p \circ q$ with respect to $v\left(\zeta^{n+1}, \cdots, \zeta^{n+m}\right)$. 
Thus, with respect to the coordinate system $w(\zeta)$, the product function of $G$ is of class $C^{2}$ and hence $G$ is a local Lie group.

Proof of The Theorem. When $G$ has the discrete center, Lemma 5 shows that $G$ is a local Lie group.

When $G$ has the non-discrete center $N$, by Lemma $4, N$ is an abelian Lie group, and by Lemma 5 again, $G / N$ is a local Lie group. Then we can introduce a canonical coordinate of the second kind by $x_{1}^{* \lambda_{1}}, \ldots, x_{m}^{* \lambda_{m}}$, where $x_{i}^{* \lambda_{i}}(i=1,2, \cdots, m)$ are one-parameter subgroups of $G / N$. Take $x_{i}$ of $G$ from the coset $x_{i}^{*}$ for each $i$, we can easily show that the set $M=\left\{y ; y=x_{1}^{\lambda_{1}} \cdots x_{m}^{\lambda_{m}},\left|\lambda_{i}\right| \leqq 1\right\}$ satisfies the condition (3) of Lemma 6 . Consequently, by Lemma $6, G$ is a local Lie group. This completes our proof.

\section{REFERENCES}

1. Garrett Birkhoff, Analytical groups, Trans. Amer. Math. Soc. vol. 43 (1934) pp. 61-101.

2. P. A. Smith, Foundation of Lie groups, Ann. of Math. vol. 48 (1947).

3. K. Iwasawa, On some types of topological groups, Ann. of Math. vol. 50 (1949).

4. L. Pontrjagin, Topological groups, Princeton, 1939.

TOKYO INSTITUTE OF TECHNOLOGY

\section{NOTE ON A THEOREM OF KOKSMA}

\section{WM. J. LEVEQUE ${ }^{1}$}

In 1935 Koksma [2] $]^{2}$ showed, among other things, that the sequence $x, x^{2}, x^{3}, \cdots$ is uniformly distributed $(\bmod 1)$ for almost all $x>1$; that is, that if $N(n, \alpha, \beta, x)$ denotes the number of elements $x^{j}$ of the sequence $x, x^{2}, \cdots, x^{n}$ for which

$$
0 \leqq \alpha \leqq x^{j}-\left[x^{j}\right]<\beta \leqq 1,
$$

then

$$
\lim _{n \rightarrow \infty} \frac{N(n, \alpha, \beta, x)}{n}=\beta-\alpha
$$

Presented to the Society, September 10,1948, under the title $A$ metric theorem on uniform distribution (mod 1); received by the editors January 24, 1949 and, in revised form, February 10, 1949.

1 The author is indebted to Professor Mark Kac for his help in connection with this paper.

2 Numbers in brackets refer to the bibliography at the end of the paper. 